\title{
Double oxide shell layer formed on a metal nanoparticle as revealed by aberration corrected (scanning) transmission electron microscopy
}

Robert Boyd, Iris Pilch, Magnus Garbrecht, M Halvarsson and Ulf Helmersson

Journal Article

\section{Tweet}

N.B.: When citing this work, cite the original article.

Original Publication:

Robert Boyd, Iris Pilch, Magnus Garbrecht and M Halvarsson, Double oxide shell layer formed on a metal nanoparticle as revealed by aberration corrected (scanning) transmission electron microscopy, Materials Research Express, 2014. 1(2), 025016

http://dx.doi.org/10.1088/2053-1591/1/2/025016

Copyright: IOP Publishing: Hybrid Open Access

http://www.iop.org/

Postprint available at: Linköping University Electronic Press

http://urn.kb.se/resolve?urn=urn:nbn:se:liu:diva-134760

I..0 


\title{
Double oxide shell layer formed on a metal nanoparticle as revealed by aberration corrected (scanning) transmission electron microscopy.
}

\author{
Robert D Boyd ${ }^{1}$, I Pilch ${ }^{1}$, M Garbrecht ${ }^{2}$, M Halvarsson ${ }^{3}$ and U Helmersson ${ }^{1}$. \\ 1. Plasma and Coatings Physics Division, IFM-Materials Physics, Linköping \\ University, SE-581 83 Linköping, Sweden. \\ 2. Thin Film Physics Division, IFM-Materials Physics, Linköping University, SE- \\ 58183 Linköping, Sweden. \\ 3. Department of Applied Physics, Chalmers University of Technology, SE-41296 \\ Göteborg, Sweden
}

E-mail: robbo@ifm.liu.se

\begin{abstract}
Determination of the extent of oxidation in batches of metal nanoparticle is essential to predict the behaviour of the material. Using aberration corrected transmission electron microscopy (TEM) it was possible to detect the formation of an oxide shell, of thickness $3 \mathrm{~nm}$, on the surface of copper nanoparticles. Further analysis shows that this shell actually consists of two layers, both of which were polycrystalline in nature with domains in the size range of 1$2 \mathrm{~nm}$, and having a thickness of $1.5 \mathrm{~nm}$ each. Energy dispersive X-ray spectroscopy confirms that the layers arise due to the formation of oxides, but it was not possible to determine their exact nature. Analysis of the intensity variation within images obtained via probe corrected scanning TEM combined with a high angle annular dark field detector indicates that the shell consists of an inner layer of cuprous oxide $\left(\mathrm{Cu}_{2} \mathrm{O}\right)$ and an outer layer of cupric oxide $(\mathrm{CuO})$. This work was complemented by conventional TEM which provided size distribution and revealed that the majority of particles have a core consisting of a single crystal of copper. This demonstrates the ability of TEM to determine the extent of oxidation of nanoparticles and its potential to be applied to a wide range of homogenous and heterogeneous nanoparticles.
\end{abstract}

Keywords: Corrected TEM, HAADF, nanoparticles, core-shell, oxide layer. 


\section{Introduction}

Engineered nanoparticles started to become commercially available in the 1970s [1] since then their potential in forming the basis of new and improved materials has become well established [2-6]. The size distribution of nanoparticle production batches is regarded as the key determinant in understanding their properties (e.g. optical, electrical and catalytic) [7] and the ability to measure this with high accuracy and precision using electron [8,9] and scanning probe [10]microscopies is now well established. However, other factors will also strongly affect their behaviour. These include shape, crystal structure, composition and the presence of a shell. This is particularly true for metal nanoparticles, consisting of materials such as copper, titanium and molybdenum (which are now becoming commercially available for a range of applications [11]) as they will oxidise in air. The formation of an oxide layer or even complete oxidation of the nanoparticle may significantly affect their properties to the detriment of their intended function. The oxidation mechanism for bulk metal have long been studied [12] and can be described by the Cabrea-Mott theory with a rapid initial growth rate which then virtually stops [13]. Both recent experimental [14,15] and theoretical [16] work on the oxidation of metal nanoparticles have indicated that this theory cannot completely describe the behaviour seen and the interpretation of the various detailed measurements is far from straightforward. A method to determine the extent of oxidation and the exact structure of the oxide layer (composition and crystal structure) is needed, along with the size distribution, in order to predict the overall properties of manufactured metal nanoparticle batches.

TEM, first developed in the 1930s [17], can routinely provide images with resolution of better than $0.2 \mathrm{~nm}$ and has been used extensively in the study of nanoparticles [10] . The closely related technique of scanning transmission electron microscopy (STEM) has also been used, especially when combined with a high angle annular dark field (HAADF) detector [18]. HAADF removes the influence of Bragg diffraction on image contrast, which is then primarily affected by the specimens atomic number, Z, and thickness [19,20]. STEM HAADF has been used to determine the shape characteristics of nanomaterials such as gold nanorods [21]. In the past decade aberration corrected instruments have become commercially available which partially remove the effect of spherical aberrations within the electron lens from the recorded image, from the objective lens for image corrected TEM and from the probe forming system in probe corrected STEM, and can routinely improve the point resolution to $0.07 \mathrm{~nm}$ [22]. Inert metal nanoparticles, such as gold [23], platinum [24] and silver [25] have been extensively studied by TEM. Their inertness prevents them from forming oxidised structures, whilst this makes them ideal for analysis by TEM their cost means they will have only limited practical applications. Several authors utilizing conventional TEM to study copper, aluminium [26] and zinc [14] nanoparticles have reported the formation of a oxide shell which can grow at elevated temperatures, to eventually form completely oxidised and hollow particles. 
Here it will be demonstrated, how the capabilities of conventional and aberration corrected TEM along with probe corrected STEM can be exploited in the characterisation of copper nanoparticles, in particular in revealing the structure of the oxide shell. In part this will be achieved using a novel method for nanoparticle production [27], which can generate high quality nanoparticles in the gas phase that can be deposited directly onto a carbon film coated TEM grid. This helps to overcome various issues with regard to the sample preparation of nanoparticles for TEM analysis. Nanoparticles prepared in a fluid have to be stabilised with surfactants and/or salts, which can contaminate the particles during the sample preparation stage. Alternatively, if the nanoparticles are stored as a powder there is a strong chance that the particles will agglomerate.

\section{Experimental}

\subsection{Nanoparticle Preparation}

All nanoparticles were prepared using a high power pulsed hollow cathode technique [27]. Gas is flowed through the hollow copper cathode so that a plasma discharge is formed. Material is the spluttered from the cathode by the plasma, which can coalesce into particles. As it does so the particles gain a negative charge which effectively controls their size. Deposition of the particles is controlled by a anode ring. The hollow cathode was driven with a pulsed power supply. For the TEM analysis two samples were prepared at different parameters: sample (1) frequency $f=700 \mathrm{~Hz}$, peak current $I_{H}=3 \mathrm{~A}\left(U_{H}=496 \mathrm{~V}\right)$, constant average power $P_{a v}=12.74 \mathrm{~W}$, deposition time $t_{D}=1 \mathrm{~min}$ and sample (2) $f=500 \mathrm{~Hz}$, peak current $I_{H}=3 \mathrm{~A}\left(U_{H}=520 \mathrm{~V}\right), P_{a v}=9.15 \mathrm{~W}$, deposition time $t_{D}=3$ $\min$. The pulse width $\left(t_{P W}=30 \mu \mathrm{s}\right)$ and the argon pressure $(P=106 \mathrm{~Pa})$ were the same. Oxidation of the particles is expected to occur outside of the deposition chamber. The particles were deposited directly onto gold TEM grids coated with a thin film of amorphous carbon (Ted Pella) and were analysed over three months and their morphology appeared not to change over this period of time.

\section{2 (Scanning) transmission electron microscopy.}

All conventional TEM images were obtained in bright field using a FEI Tecnai $\mathrm{G}^{2}$ TF20 UT microscope equipped with a field emission gun operating at a voltage of $200 \mathrm{kV}$. High resolution aberration corrected (S)TEM images were performed using the Linköping monochromated doublecorrected FEI $\operatorname{Titan}^{3}$ 60-300 instrument operated at $300 \mathrm{kV}$. Additional STEM images were obtained with a monochromated probe corrected FEI Titan 80-300 instrument. Both microscopes exhibits a 
high brightness Schottky-type field emission gun (XFEG). Energy dispersive X-ray spectroscopy (EDS) measurements were taken using the FEI Titan 80-300 instrument and Oxford Instrument INCA system and line profiles were calculated with the background subtracted.

\section{Results and Discussion}

First conventional bright field TEM imaging was undertaken, to characterise the particles and their size distribution. They were first imaged at relatively low resolution with a small objective aperture $(20 \mu \mathrm{m})$. This has the effect of enhancing the contrast, with an example image shown in Figure 1(a). Individual particles are clearly visible and are dispersed across the carbon film. The size distribution of the particles could then be easily measured using automatic image analysis [28] using the ImageJ software package [29]. The maximum Feret measurement was used [30] and the size distribution from the two samples is shown in Figure 1(b). Both samples are highly monodispersed with a narrow size distribution with Sample 2 having a smaller average particle size, $(23.0 \pm 9.6 \mathrm{~nm}$ compared to $26.4 \pm$ $8.9 \mathrm{~nm}$ ). Both samples are seen to show an addition and smaller mode in their size distribution at a diameter of $7 \mathrm{~nm}$. From the low resolution images a possible shell layer was seen on all of the particles. High resolution bright field transmission electron microscopy imaging shows that in the majority of cases that the particles have a core consisting of a single crystal (Figure 1(c)). Although a significant minority of particles do show a core consisting of polycrystalline domains. An example of this is shown in Figure 1(d) where the core consists of two domains with a grain boundary between them. The shell layer, apparent in the low resolution images could not be clearly resolved in the HRTEM images, in part because of the well-known phenomenon of delocalization of the lattice fringes due to aberrations within the objective lens [31].

Partially removing the effect of these aberrations by, via the use of a image corrected TEM instrument, is then required to perform a more detailed study of the nanoparticle structure and in particular of its shell. Apart from the size distribution, both samples appear identical and the results of analysis for both samples of nanoparticles are subsequently combined. An aberration corrected high resolution TEM image of a single nanoparticle is shown in Figure 2(a) clearly showing a core and a shell layer and its fast Fourier transform (FFT) is shown in Figure 2(b). The higher information limit of the corrected instrument compared to conventional TEM is clearly observable from this pattern showing additional features (spots) corresponding to higher spatial frequencies as compared to the conventional TEM image's FFT (Figure 1(c)). This diffractogram was indexed to copper as indicated in Figure 2(b). Also apparent in the FFT pattern is a ring feature with a radius of $4.1 \mathrm{~nm}^{-1}$, indicating the presence of multi-crystalline domains. Inversing the FFT pattern excluding the spots due to the copper metal 
reveals that the core consists of a single crystal of copper (Figure 2(c)) whilst the shell is polycrystalline in nature. Indeed the shell appears to consist of two distinct layers both with crystalline domains 1- $2 \mathrm{~nm}$ in size with a different crystal structure (Figure 2(d)). However for these nano-sized crystallites, the number of lattice planes imaged was too small and their orientation unknown meaning that their identification was not possible.

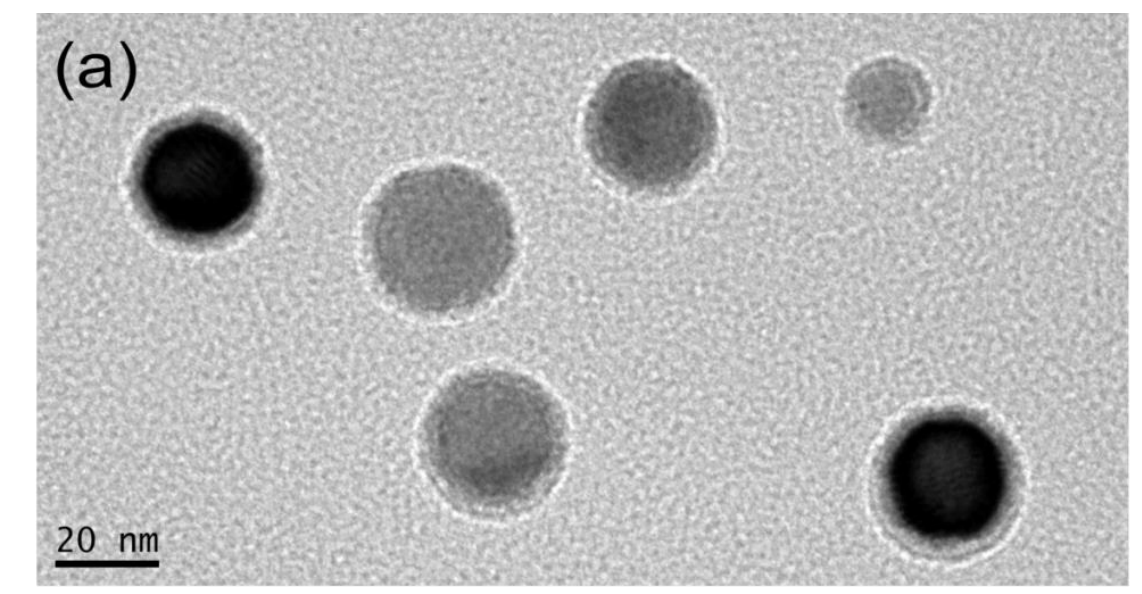

(b)
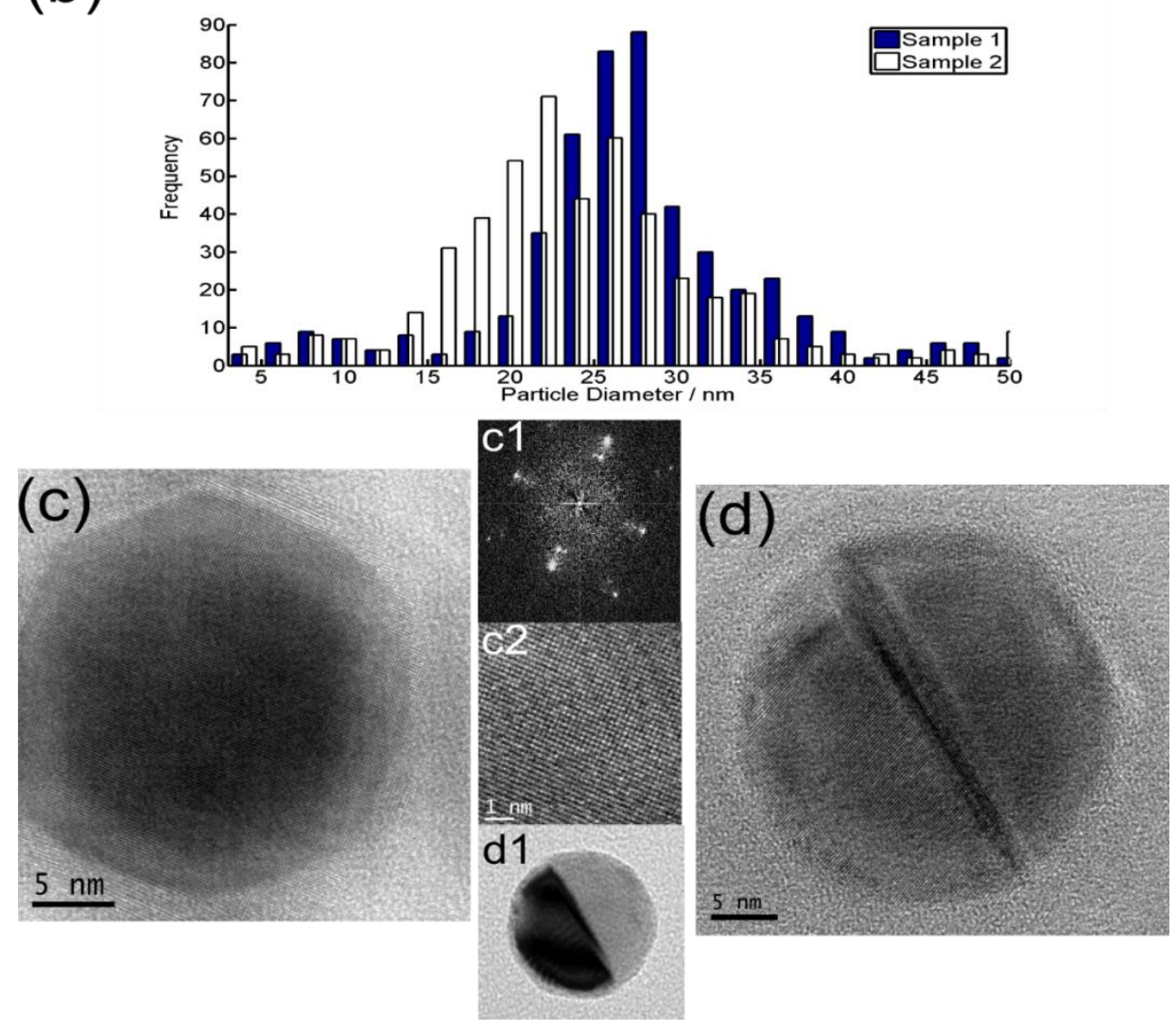

Figure 1: Transmission electron microscopy analysis of copper nanoparticles using a conventional instrument. (a) A typical low resolution image of copper nanoparticles showing a core / shell structure.

(b) The obtained size distributions from low resolution images for the two samples. Typical high resolution TEM image from a particles with (c) a single crystalline core (c1: FFT pattern and $\mathrm{c} 2$ : zoomed in on the central core area of the nanoparticle) and (d) a poly crystalline core, with two main domains (d1: same particle imaged at low resolution with a small objective aperture). 


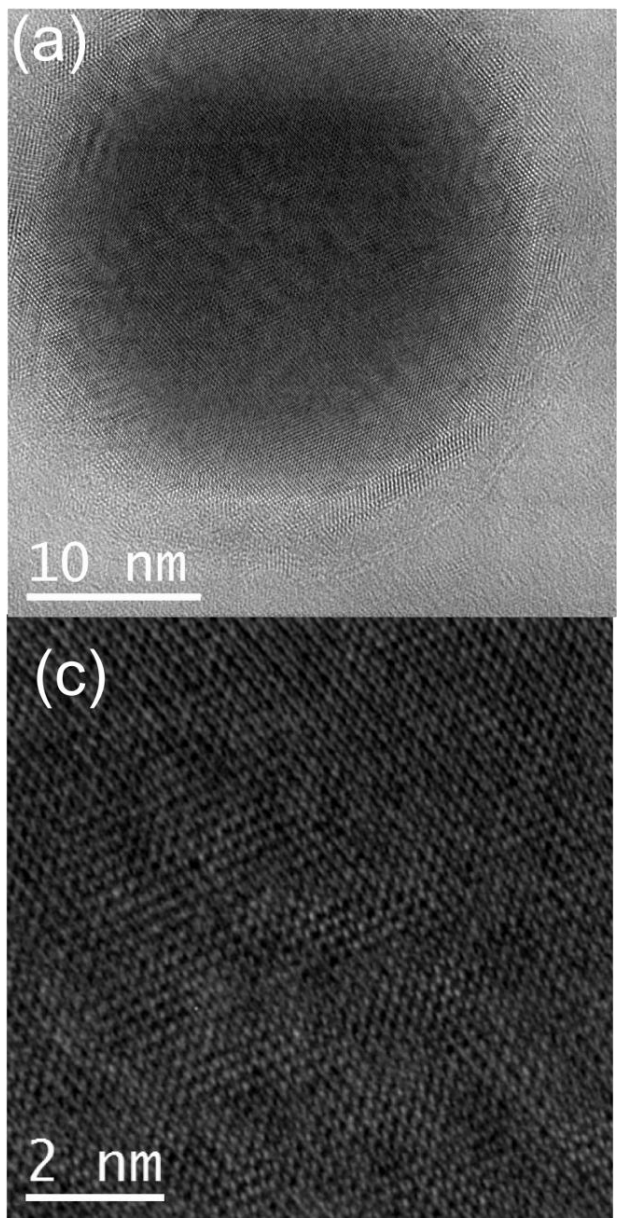

\section{(b)}
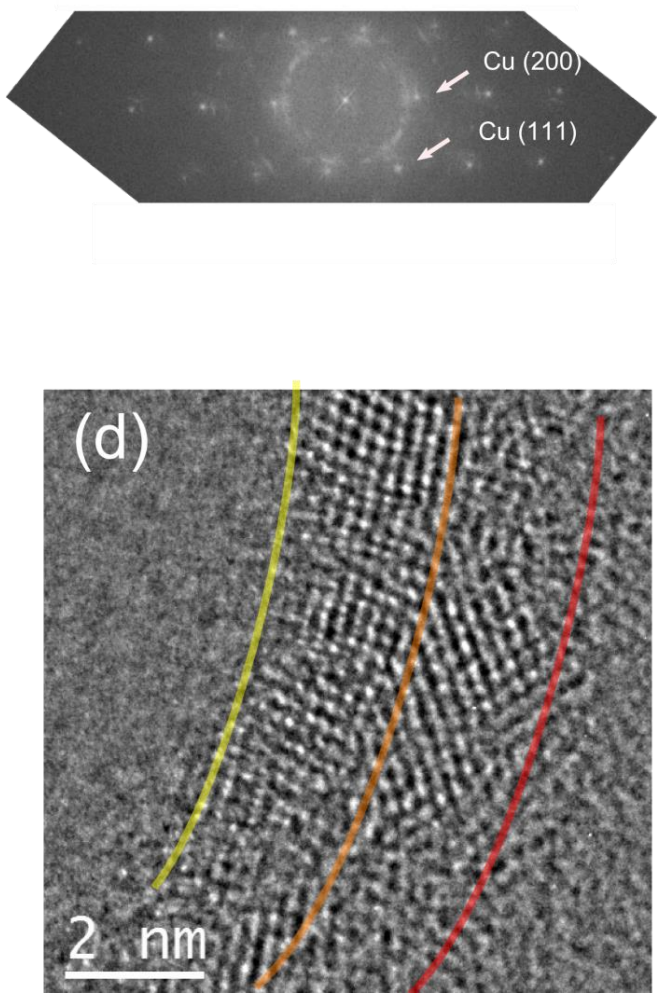

Figure 2: Analysis of the copper particles using aberration corrected transmission electron microscopy. (a) noise filtered high resolution image and (b) its corresponding fast Fourier transform. Zoomed in images of the particles (c) central core and (d) surface, the latter obtained by inverting the FFT pattern shown in (b) with the spot pattern filtered out. (with the approximate positions of the shell layers highlighted)..

The structure of the core and shell was further examined using probe corrected scanning transmission electron microscopy and the results are summarized in Figure 3, both bright field and HAADF imaging were used to image particles simultaneously. Low resolution bright field and HAADF images are shown in Figures 3(a) and (b) respectively. Both confirm the presence of particles consisting of a core and a shell. From these images the typical thickness of the shell could be measured $(3 \mathrm{~nm})$ and, due to its darker appearance in the HAADF images, has a lower overall atomic mass compared to the core. Increasing the magnification (Figure 3(c)) and the HAADF image again reveals the bi-layer nature of the shell, with inner and outer layers of thickness $1.5 \mathrm{~nm}$. A high resolution HAADF image is shown in Figure 3(d) and zooming on the shell (Figures 3(e and f)) again shows the single crystalline core surrounded by multi crystalline domains. Further examples are given in the supporting information. EDS reveals the presence of copper and oxygen, which could be directly associated with the particles. An EDS line scan across a single particle is shown in Figure 3(g) showing the copper and oxygen signals. If we assume that the particle consists of a copper core surrounded by an oxidised shell, of thickness $3 \mathrm{~nm}$, then a first approximation of the EDS line scans can be generated. This was 
done by normalising the line scans with respect to the thickness variation for a hollow sphere, excluding the free space, for the oxygen signal and a solid sphere for the copper signal with a correction to reduce the signal from the shell region due to the presence of oxygen. The predicted copper and oxygen line profiles can then be plotted as also shown in Figure 3(g). There is a good correlation between the actual and predicted line profile. Especially the oxygen signal, which is seen to peak then drop to $50 \%$ of its maximum value as it moves towards the centre of the particle which is predicted by the oxide shell model. Whilst this confirms the presence of an oxide shell on the particles, the EDS signal is too low to determine its exact nature and composition.
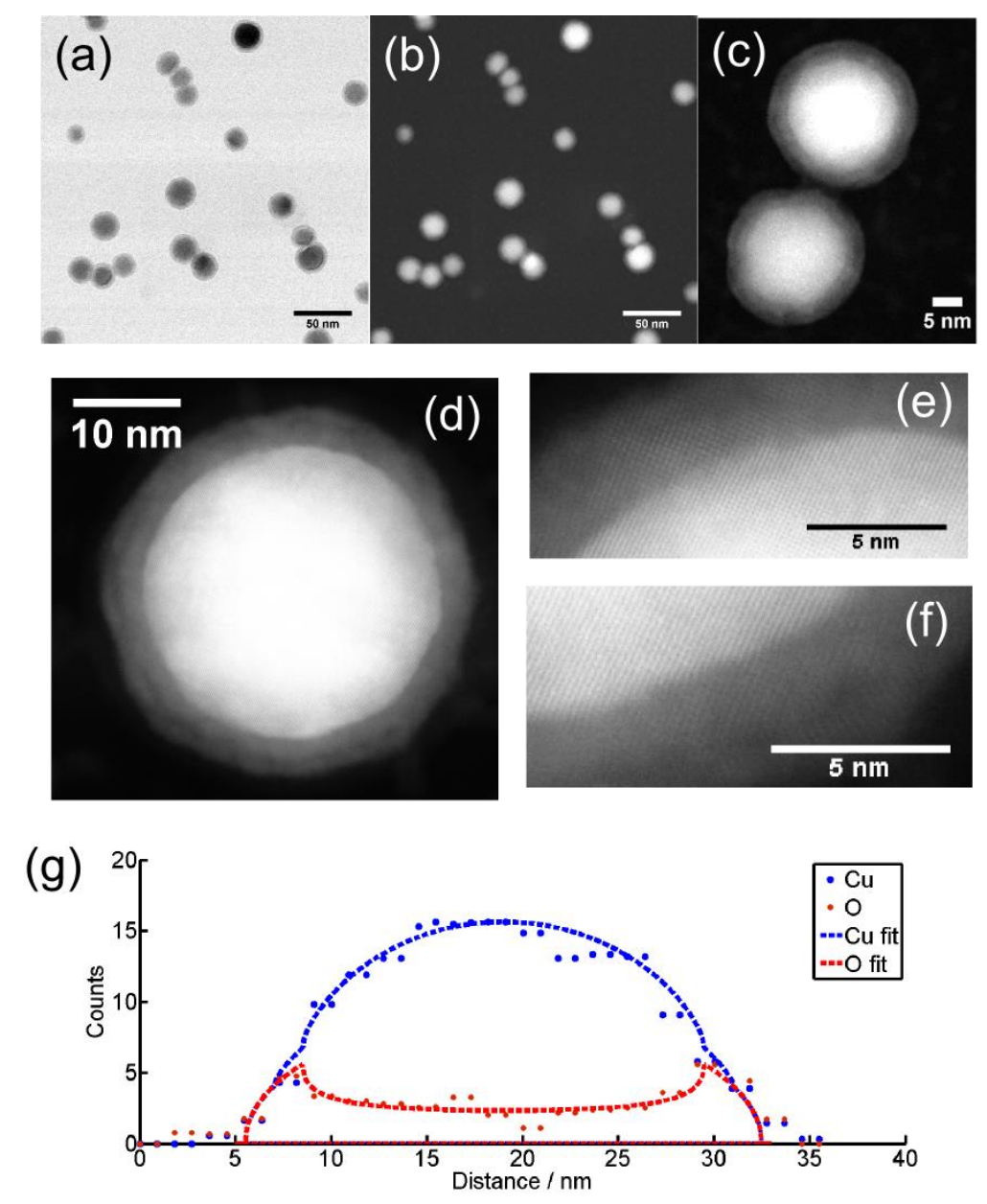

Figure 3: Analysis of the copper nanoparticles using probe corrected transmission electron microscopy. Low resolution images using the (a) bright field and (b) high angle annular dark field (HAADF) detectors; both are showing a shell structure surrounding the particles. (c) Higher resolution using the HAADF detector with the bi-layer nature of the shell visible. (d-f) High resolution HAADF image of the particle showing a single crystal core and the poly-crystalline nature of the shell layer and

(g) EDS line scan across a single particle showing the copper and oxygen signal along with the predicted line profiles assuming a metal core and a oxidised shell. 
All the results are consistent with the particles consisting of a core of copper surrounded by a thin shell of oxidised materials. This shell actually consists of two layers both of which are polycrystalline in nature. Copper is known to form two types of oxide, cuprous oxide $\left(\mathrm{Cu}_{2} \mathrm{O}\right)$ and cupric oxide $(\mathrm{CuO})$. As is common with many materials the oxide layers are too fragile for extensive investigations using the analytical methods of EDS and electron energy loss spectroscopy. In order to try and determine the exact nature of these shell layers for the STEM investigation, further analysis of the probe corrected HAADF-STEM images was undertaken by deriving HAADF images using a first approximation based on a geometric model. This was done by assuming that both the intensity contrast (I) in the HAADF image is dependent primarily on the sample thickness $(\mathrm{t})$ and the mean atomic number $(\mathrm{Z})$ via the relationship [32]

$$
I=f\left(t, Z^{1.7}\right) .
$$

and that the particle could be represented by a simple geometric model [33] for the particles with the core being modelled by an anisotropic ovoid (non-spherical factor of 0.8). Firstly a HAADF image of a particle was selected (Figure 4(a and b) reproduced from Figure 3(c)). Note that this particle neither has a crystallographic zone axis aligned with the electron beam nor any apparent defects within the core Having the crystalline zone axis aligned to the electron beam [34] and the presences of defects[35] can both affect the HAADF image intensity. From these assumptions a geometric model of the particle is produced (Figure 4(c)) and finally a derived HAADF image (Figure 4(d)). The model image appears similar to the actual HAADF image. Taking a cross section from the HAADF image (Figure 5(a)) and comparing to the derived intensities (Figure 5(b)) shows that the closest fit is for a copper core surrounded by a shell with an inner layer of $\mathrm{Cu}_{2} \mathrm{O}$ and an outer layer of $\mathrm{CuO}$. Repeating this fitting to a different particle is provided in the supplementary information Figure S2.

Previously the oxide layer on copper nanoparticles has been seen as a continuous shell [26]. This is the first time that the shell has been revealed to have two layers, with different oxidation states, consisting of polycrystalline domains. 


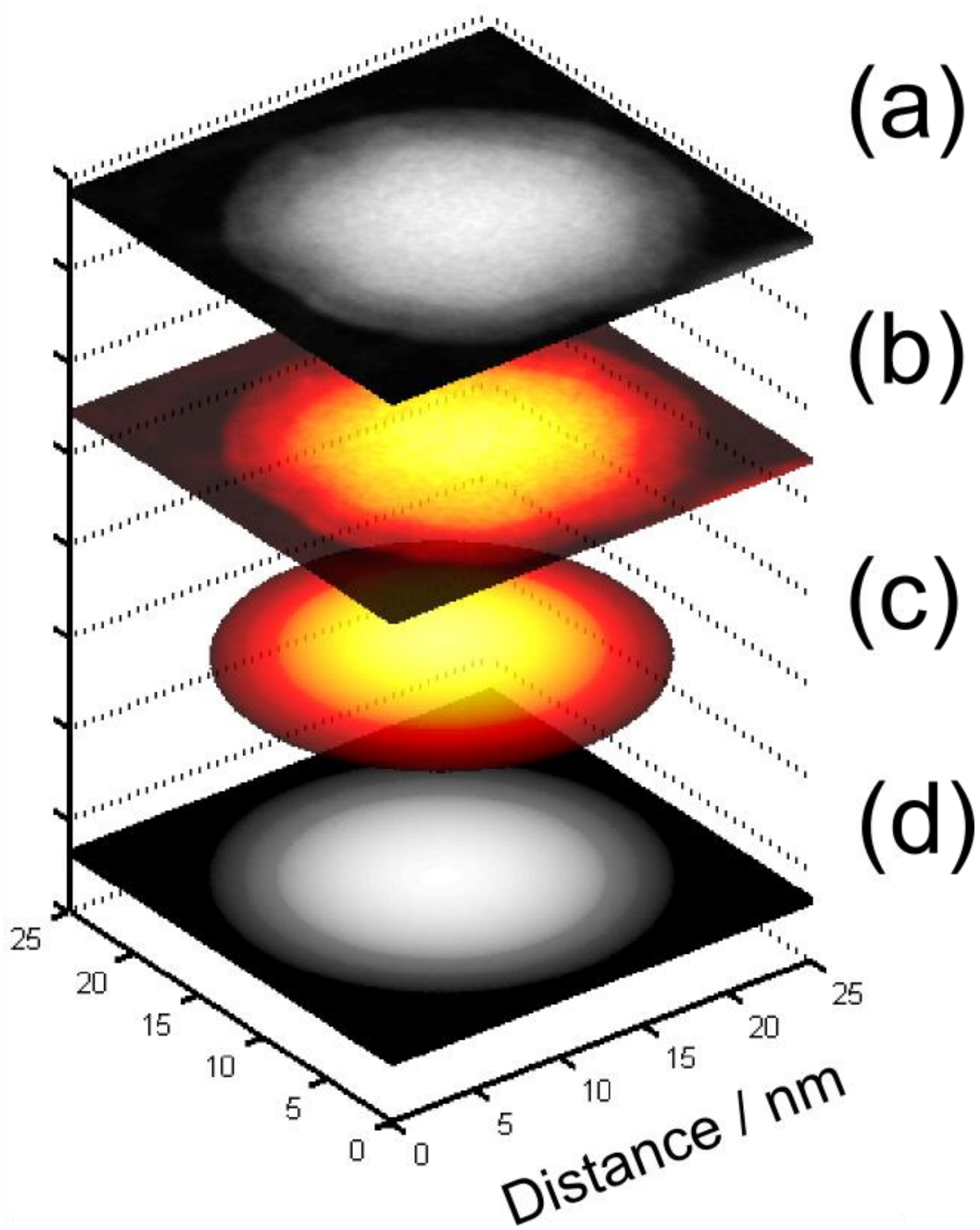

Figure 4: Stack plot showing the analysis of the HAADF image. (a) measured HAADF image of a single nanoparticle. (b) The same HAADF image artificially coloured to highlight the core and the two layers of the shell (c) The geometric representation of the nanoparticle and (d) the resulting derived HAADF image based on (c). 

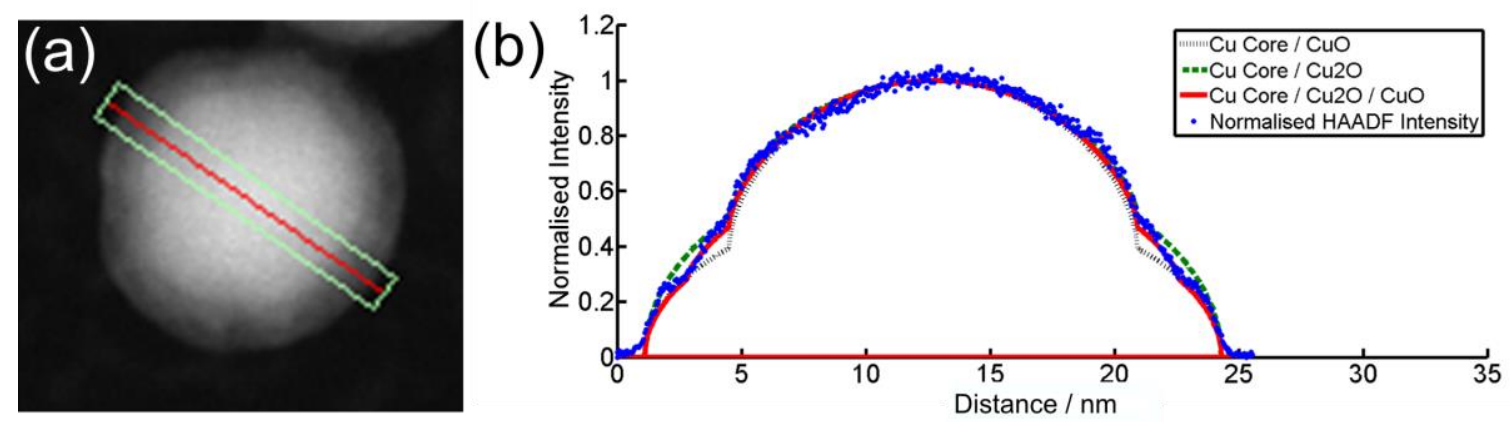

Figure 5. (a) HAADF image of the nanoparticle used for intensity comparison. The red line indicates the central location of the intensity profile as averaged over a region defined by the green rectangle. (b) Comparison of the intensity profiles produced from the different geometric representations of the coreshell nanoparticle with the actual normalised HAADF intensity.

\section{Conclusions}

Corrected scanning and bright field transmission electron microscopy has revealed the formation of nanometre sized oxide crystals on the surface of copper nanoparticles. These form a bilayer shell, 3 $\mathrm{nm}$ thick, with different compositions for each layer. Oxide layers forming on metal nanoparticles could strongly affect their behaviour. The approach highlighted here demonstrates how these can be effectively studied and also has applications for other heterogeneous nanomaterials.

\section{References}

[1] Granqvist C G and Buhrman R A 1976 Ultrafine metal particles J. Appl. Phys. 47 2200-19

[2] Jennings T and Strouse G 2007 Past, Present, and Future of Gold Nanoparticles Adv. Exp. Med. Biol. $62034-47$

[3] Shipway A N, Katz E and Willner I 2000 Nanoparticle Arrays on Surfaces for Electronic, Optical, and Sensor Applications ChemPhysChem 1 18-52

[4] Makise K, Mitsuishi K, Kokubo N, Yamaguchi T, Shinozaki B, Yano K, Inoue K and Nakamura H 2010 Transport properties and microstructures of polycrystalline $\mathrm{In}$ [sub 2]O[sub 3]-ZnO thin films J. Appl. Phys. 108023704

[5] Nozik A J, Beard M C, Luther J M, Law M, Ellingson R J and Johnson J C 2010 Semiconductor Quantum Dots and Quantum Dot Arrays and Applications of Multiple Exciton Generation to Third-Generation Photovoltaic Solar Cells Chem. Rev. 110 6873-90 
[6] Raimondi F, Scherer G G, Kötz R and Wokaun A 2005 Nanoparticles in Energy Technology: Examples from Electrochemistry and Catalysis Angew. Chem. Int. Ed. 44 2190-209

[7] Burda C, Chen X, Narayanan R and El-Sayed M A 2005 Chemistry and Properties of Nanocrystals of Different Shapes Chem. Rev. 105 1025-102

[8] Meli F, Klein T, Buhr E, Frase C G, Gleber G, Krumrey M, Duta A, Duta S, Korpelainen V, Bellotti R, Picotto G B, Boyd R D and Cuenat A 2012 Traceable size determination of nanoparticles, a comparison among European metrology institutes Meas. Sci. Technol. 23 125005

[9] Boyd R D, Cuenat A, Meli F, Klein T, Frase C G, Gleber G, Krumrey M, Duta A, Duta S, Hogstrom R and Prieto E 2011 Measurement Good Practice Guide No.119; Good Practice Guide for the Determination of the Size and Size Distribution of Spherical Nanoparticle samples

[10] Boyd R D and Cuenat A 2011 New image analysis procedure for fast and reliable size measurement of nanoparticles from scanning probe microscopy images J. Nanoparticle Res. $\mathbf{1 3}$ $105-13$

[11] Narayanan R and El-Sayed M A 2005 Catalysis with Transition Metal Nanoparticles in Colloidal Solution: Nanoparticle Shape Dependence and Stability J. Phys. Chem. B 10912663 76

[12] Rabald 1954 Oxidation of metals and alloys. Von O. Kubaschewski und B. E. Hopkins. London: Butterworths Scientific Publications 1953. 239 S., 84 Abb., 18 Zahlentafeln, 35 Mater. Corros. 5 477-477

[13] Cabrera N and Mott N F 1949 Theory of the oxidation of metals Rep. Prog. Phys. 12163

[14] Mahapatra A K, Bhatta U M and Som T 2012 Oxidation mechanism in metal nanoclusters: Zn nanoclusters to ZnO hollow nanoclusters J. Phys. Appl. Phys. 45415303

[15] Nakamura R, Lee J-G, Tokozakura D, Mori H and Nakajima H 2007 Formation of hollow ZnO through low-temperature oxidation of Zn nanoparticles Mater. Lett. 61 1060-3

[16] Zhdanov V P and Kasemo B 2008 Cabrera-Mott kinetics of oxidation of nm-sized metal particles Chem. Phys. Lett. 452 285-8

[17] Goodhew P 1988 Electron microscopy and analysis (London ;NNew York: Taylor \& Francis)

[18] Mayoral A, Deepak F L, Esparza R, Casillas G, Magen C, Perez-Tijerina E and Jose-Yacaman M 2012 On the structure of bimetallic noble metal nanoparticles as revealed by aberration corrected scanning transmission electron microscopy (STEM) Micron 43 557-64

[19] Grillo V, Carlino E, Ciasca G, Seta M D and Ferrari C On the Role of Specimen Thickness in Chemistry Quantification by HAADF Microscopy of Semiconducting Materials $2007 \mathrm{vol}$ 120, ed A G Cullis and P A Midgley (Dordrecht: Springer Netherlands) pp 173-6

[20] Van Aert S, Batenburg K J, Rossell M D, Erni R and Van Tendeloo G 2011 Three-dimensional atomic imaging of crystalline nanoparticles Nature 470 374-7

[21] Boyd R D, Young T J and Stolojan V 2012 Characterisation of gold nanoparticles and rods using high angle annular dark field imaging J. Nanoparticle Res. 14

[22] Lentzen M, Jahnen B, Jia C L, Thust A, Tillmann K and Urban K 2002 High-resolution imaging with an aberration-corrected transmission electron microscope Ultramicroscopy 92 233-42 
[23] Katz-Boon H, Rossouw C J, Weyland M, Funston A M, Mulvaney P and Etheridge J 2011 Three-Dimensional Morphology and Crystallography of Gold Nanorods Nano Lett. 11 273-8

[24] Chang L Y, Barnard A S, Gontard L C and Dunin-Borkowski R E 2010 Resolving the Structure of Active Sites on Platinum Catalytic Nanoparticles Nano Lett. 10 3073-6

[25] Scholl J A, Koh A L and Dionne J A 2012 Quantum plasmon resonances of individual metallic nanoparticles Nature $\mathbf{4 8 3} 421-7$

[26] Nakamura R, Tokozakura D, Nakajima H, Lee J-G and Mori H 2007 Hollow oxide formation by oxidation of $\mathrm{Al}$ and $\mathrm{Cu}$ nanoparticles J. Appl. Phys. 101074303

[27] Pilch I, Söderström D, Brenning N and Helmersson U 2013 Size-controlled growth of nanoparticles in a highly ionized pulsed plasma Appl. Phys. Lett. 102 033108-033108-4

[28] Reetz M T, Maase M, Schilling T and Tesche B 2000 Computer image processing of transmission electron micrograph pictures as a fast and reliable tool to analyze the size of nanoparticles J. Phys. Chem. B 104 8779-81

[29] Abramoff M, Magelhaes P and Ram S 2004 Image Processing with ImageJ Biophotonics Int. 11 $36-42$

[30] Allen T 1999 Particle size measurement. (Dordrecht: Kluwer Academic Publishers)

[31] Williams D and Carter C B 2009 Transmission electron microscopy (New York [u.a.]: Plenum Press)

[32] Howie A 1979 Image Contrast And Localized Signal Selection Techniques J. Microsc. $11711-$ 23

[33] Li Z Y, Wilcoxon J P, Yin F, Chen Y, Palmer R E and Johnston R L 2008 Structures and optical properties of 4-5 nm bimetallic AgAu nanoparticles Faraday Discuss. 138363

[34] Yu Z, Muller D A and Silcox J 2008 Effects of specimen tilt in ADF-STEM imaging of a-Si/cSi interfaces Ultramicroscopy 108 494-501

[35] Lupini A R, Borisevich A Y, Idrobo J C, Christen H M, Biegalski M and Pennycook S J 2009 Characterizing the Two- and Three-Dimensional Resolution of an Improved AberrationCorrected STEM Microsc. Microanal. 15441 


\section{Supporting information S1:}

Bright (a) and HAADF (b) high resolution images of the same nanoparticles
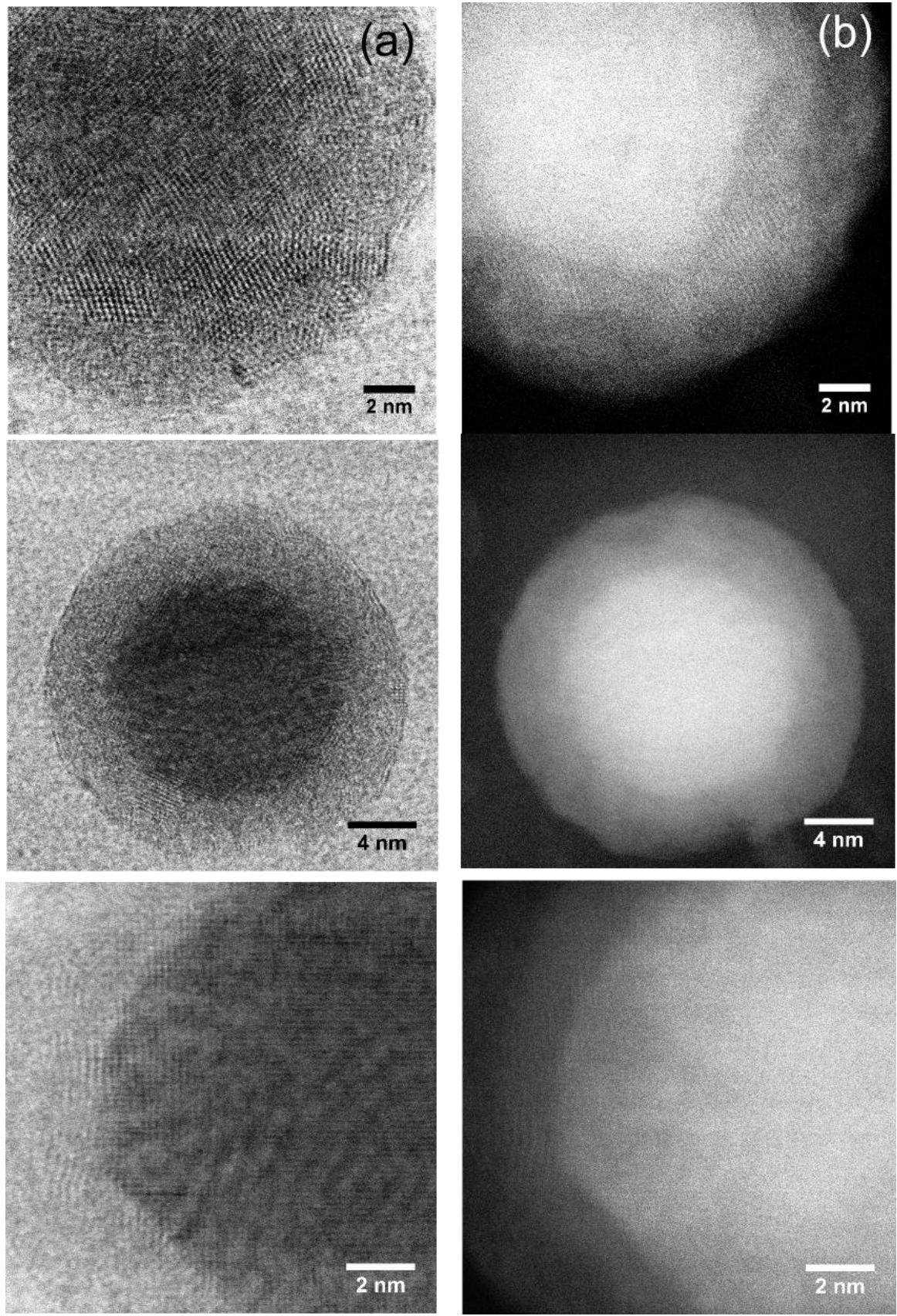

This is also revealed in the bright field and HAADF images shown in figures 3 ( $g$ and $h$ ) respectively, where the bright field image again shows the crystalline regions extending to the end of the nanoparticle whilst the HAADF image reveals the shape and location of the core and shell. 
S2: Additional analysis of a different particle. Information as per text
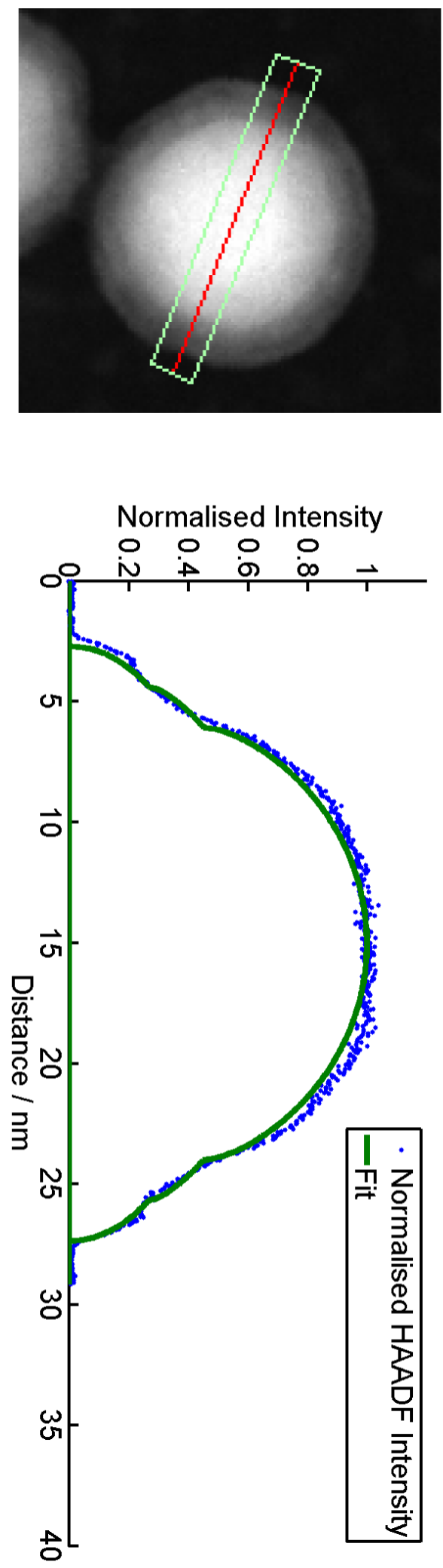